P-204 BISPHOSPHONATE TREATMENT IN BONE METASTASES: AN INTERVENTIONAL OUTPATIENT CLINIC MODEL

${ }^{1}$ Kathe'rine Newton, ${ }^{1}$ Ruth Keeble, ${ }^{1,2}$ Nikki Reed. 'Marie Curie Hospice, West Midlands, Soilhull, UK; ${ }^{2}$ Heart of England NHS Foundation Trust, Birmingham, UK

10.1136/bmjspcare-2016-001245.225

Background Patients with bone metastases can suffer from considerable pain and reduced quality of life, in addition to complications such as pathological fracture and spinal cord compression. Bisphosphonates can be used as an adjunct to treat pain in addition to conventional analgesia and radiotherapy.

Aims Our outpatient bisphosphonate infusion service at the hospice was set up in 2008, in conjunction with our local acute NHS trust oncology unit. The pilot service initially accepted patients with bone metastases from hormone refractory prostate cancer when pain was not controlled with conventional radiotherapy and analgesia.

Method Patients benefit from an outpatient appointment with a doctor and nurse to assess current symptoms using IPOS (Integrated Palliative care Outcome Scale), followed by bisphosphonate treatment on the same day. Referral into other services within the hospice, such as physiotherapy, occupational therapy, rehab services and our FAB (Fatigue, Anxiety and Breathlessness) clinic, can be made when needed.

Results Following review of the service we now accept patients earlier in their disease trajectory and treat patients with bone involvement from other diagnoses such as myeloma, sarcoma or renal cancer. Our referral numbers continue to increase on a yearly basis.

We have successfully treated patients with recurrent hypercalcaemia in the day case setting by monitoring levels closely and treating early with a bisphosphonate before they are symptomatic; thereby reducing morbidity, preventing the need for inpatient admission and reducing pressure on acute hospital services.

Conclusion Future expansion of this interventional outpatient clinic might include breast cancer patients who currently receive denosumab injections in the hospital. We could use this model of service to allow early integration of these patients into palliative care services and allow them to benefit from the diversity of therapies and services the hospice can offer at an earlier stage in their illness.

\section{P-205 HOW TO PROVIDE A VOLUNTEER BEFRIENDING SERVICE: RECOMMENDATIONS FROM A WAIT-LIST CONTROLLED TRIAL}

${ }^{1}$ Catherine Walshe, ${ }^{1}$ Steven Dodd, ${ }^{1}$ Guillermo Perez Algorta, ${ }^{2}$ Matthew Hill, ${ }^{2}$ Nick Ockenden, ${ }^{1}$ Sheila Payne, ${ }^{1}$ Nancy Preston. ${ }^{1}$ International Observatory on End of Life Care, Lancaster, UK; ${ }^{2}$ Institute for Volunteering Research, NCVO, London, UK

\subsection{6/bmjspcare-2016-001245.226}

Background Many innovations in palliative care are commenced without robust research to understand either their impact, nor explore the processes, barriers and facilitators to effective implementation of the service. Within the hospice sector there are an increasing number of volunteer befriending or good neighbour services, and evidence is needed on how best to provide these to improve outcomes.

Aim To make evidence based recommendations on how to deliver effective volunteer befriending services at the end of life.
Methods A wait-list controlled trial (ELSA) (with eight nested qualitative case studies) testing volunteer delivered befriending services across 11 hospice, charity and NHS sites. Participants were estimated to be in their last year of life, randomly allocated to receive the befriending intervention immediately or after a four week wait. Data collection at baseline, four, eight weeks assessed patient's quality of life, loneliness and social support. The case studies included in-depth qualitative interviews with staff, volunteers, patients and family carers. ISRCTN 12929812.

Results 195 people entered the trial, and interviews were conducted across eight case study sites with volunteers $(n=23)$, staff $(\mathrm{n}=31)$, patients $(\mathrm{n}=24)$ and family carers $(\mathrm{n}=3)$. Key issues include strategies for maximising impact (e.g. frequency and length of visits, type of support provided, targeting patients), the precise nature of the volunteer role (social or practical, in home or getting out and about), effective running of the service (e.g. how to match volunteer and patient, supporting volunteers), and managing the different nature of a volunteer delivered service(e.g. volunteer training, negotiating boundary issues).

Conclusions We will provide evidence based recommendations on how to run a high quality volunteer befriending or good neighbour service in an effective, safe and well managed way which is likely to maximise impact. Funded by the UK Cabinet Office. See also oral presentation on volunteer befriending services on page (A6).

\section{P-206 REACHING THE WIDER NEED - A PILOT - THE ORCHARD SUPPORTIVE CARE CLINIC}

Charlotte Williams, Glenys Le Poidevin, Bev Aplin. St Wilfrid's Hospice, Chichester, UK

\subsection{6/bmjspcare-2016-001245.227}

Hospice care continues to meet the needs of only a minority of patients who have specialist palliative care needs, either because prognosis is uncertain, patients themselves are not ready to accept 'hospice' or referrer/referee do not directly consider palliative care needs or prognosis. Indeed, many patients with non-cancer diagnoses will never see a palliative care specialist despite complex symptomatology and psychological morbidity. Recognising this gap, St Wilfrid's Hospice developed a pilot clinic to bridge a gap in provision: accepting any patient with complex physical or psychological symptoms needing specialist clinical support/advice -associated with a chronic life-limiting illness of any diagnosis (or its treatment related symptom burden.) Prognosis could exceed 12 months (unlike hospice criteria) or be unestablished/uncertain. The clinic aims were to promote proactive management- including self-management of symptoms, provide information for both patient and carers, offer an advance care planning course and breathlessness/fatigue management course, and provide general emotional support. The multidisciplinary team included a palliative care consultant, 2 CNSs, occupational and physiotherapy, a complementary/art therapy and a volunteer.

65 patients were referred ( 9 declined input, one died before seen, six were too unwell), $40 \%$ of these had non- cancer diagnoses. $60 \%$ of referrals came from secondary care (commonest specialities-respiratory, neurology and urology.) Of the 49 patients seen in OSCC, 21 were later transferred to the main hospice caseload as their illness progressed; 12 then died once under the care of the main hospice, all of whom had completed advance care planning before transfer. Patients able to self-report symptoms completed Edmonton Symptom Assessment Scores (ESAS) 